Reduction of the centroprojective connection of the projective group to the fundamental-group connection of a surface

\author{
Jury Shevchenko and Elena Skrydlova
}




\title{
REDUCTION OF THE CENTROPROJECTIVE CONNECTION OF THE PROJECTIVE GROUP TO THE FUNDAMENTAL-GROUP CONNECTION OF A SURFACE
}

\author{
JURY SHEVCHENKO AND ELENA SKRYDLOVA
}

\begin{abstract}
The projective group effectively acting in the multi-measured projective space is represented by the principal bundle of the centroprojective frames, in which a symmetrical centroprojective connection is defined. A surface is considered in the projective space. The principal bundle above the surface with the typical fiber - a subgroup of the stationarity for the centered tangent plane to the surface in a fixed point is considered. Fundamental-group connection is given in this fibering. It consists of tangent affine and centroprojective, normal linear and affinegroup connections. It is shown that the centroprojective connection in the projective group is reduced to the fundamental-group connection in the principal bundle associated with the surface in the projective space.
\end{abstract}

2000 Mathematics Subject Classification: 53A20, 53B25, 53B05, 53B10, 53B15

Keywords: Projective space, projective group, principal bundle, fundamental-group connection, affine connection, centroprojective connection, linear connection, affine-group connection.

\section{Centroprojective Connection in the Projective Group}

In $n$-dimensional projective space $P_{n}$, let us consider the moving frame $\left\{A, A_{I}\right\}$ $(I, \cdots=\overline{1, n})$ with the derivation formulas

$$
d A=\vartheta A+\theta^{I} A_{I}, \quad d A_{I}=\vartheta A_{I}+\theta_{I}^{J} A_{J}+\theta_{I} A,
$$

where the form $\vartheta$ plays the role of a proportionality factor and the structure forms $\theta^{I}, \theta_{J}^{I}, \theta_{I}$ of the projective group $\operatorname{GP}(n)$, which acts effectively in the space $P_{n}$, satisfy the Cartan equations (see, e.g., [3])

$$
\begin{aligned}
& D \theta^{I}=\theta^{J} \wedge \theta_{J}^{I}, \\
& D \theta_{J}^{I}=\theta_{J}^{K} \wedge \theta_{K}^{I}+\theta^{K} \wedge \theta_{J K}^{I}, \\
& D \theta_{I}=\theta_{I}^{J} \wedge \theta_{J} ; \\
& \theta_{J K}^{I}=-\delta_{J}^{I} \theta_{K}-\delta_{K}^{I} \theta_{J} .
\end{aligned}
$$

The equations (1.2) are the structure equations of the principal fiber bundle of the centroprojective frames $C_{n(n+1)}\left(P_{n}\right)$, whose base is the projective space $P_{n}$ (a region circumscribed by a point $A$ ). The centroprojective (coaffine) group $C_{n(n+1)}=$ 
$G A^{*}(n) \subset \mathrm{GP}(n)$ is the typical fiber. This group acts in any centroprojective space $P_{n}^{0}$ that is the space $P_{n}$ with the fixed point $A$.

Proposition 1. The fibering $C_{n(n+1)}\left(P_{n}\right)$ of the centroprojective frames has the principal quotient bundle $L_{n^{2}}\left(P_{n}\right)$ with the structure equations $\left(1.2_{1}\right),\left(1.2_{2}\right)$ the same base $P_{n}$, and typical fiber $L_{n^{2}}=\mathrm{GL}(n)$, where $\mathrm{GL}(n)$ is the linear quotient group acting [2] ineffectively in the pencil of lines passing through the point $A$ of the projective space $P_{n}$ (this group acts in the projective quotient space $P_{n-1}=P_{n} / A$ (see, e.g., [1])).

We give a centroprojective connection on the principal fiber bundle $C_{n(n+1)}\left(P_{n}\right)$ using Laptev-Lumiste method $[4,6,8]$ by the forms

$$
\hat{\theta}_{J}^{I}=\theta_{J}^{I}-\Pi_{J K}^{I} \theta^{K}, \quad \hat{\theta}_{I}=\theta_{I}-\Pi_{I J} \theta^{J},
$$

where the components of the centroprojective connection object $\Pi=\left\{\Pi_{J K}^{I}, \Pi_{I J}\right\}$ satisfy the differential equations

$$
\begin{aligned}
\Delta \Pi_{J K}^{I}+\theta_{J K}^{I} & =\Pi_{J K L}^{I} \theta^{L}, \\
\Delta \Pi_{I J}+\Pi_{I J}^{K} \theta_{K} & =\Pi_{I J K} \theta^{K} .
\end{aligned}
$$

The tensor operator $\Delta$ acts by

$$
\Delta \Pi_{J K}^{I}=d \Pi_{J K}^{I}+\Pi_{J K}^{L} \theta_{L}^{I}-\Pi_{L K}^{I} \theta_{J}^{L}-\Pi_{J L}^{I} \theta_{K}^{L} .
$$

Proposition 2. The centroprojective connection object $\Pi$ is a quasitensor containing the quasitensor $\Pi_{J K}^{I}$ which determines an affine (special linear) connection on the fibering $L_{n^{2}}\left(P_{n}\right)$ of linear frames.

The forms of centroprojective connection (1.4) satisfy the structure equations

$$
\begin{aligned}
D \hat{\theta}_{J}^{I} & =\hat{\theta}_{J}^{K} \wedge \hat{\theta}_{K}^{I}+K_{J K L}^{I} \theta^{K} \wedge \theta^{L}, \\
D \hat{\theta}_{I} & =\hat{\theta}_{I}^{J} \wedge \hat{\theta}_{J}+K_{I J K} \theta^{J} \wedge \theta^{K} .
\end{aligned}
$$

The components of the centroprojective curvature object $K=\left\{K_{J K L}^{I}, K_{I J K}\right\}$ are defined by

$$
K_{J K L}^{I}=\Pi_{J[K L]}^{I}-\Pi_{J[K}^{M} \Pi_{M L]}^{I}, \quad K_{I J K}=\Pi_{I[J K]}-\Pi_{I[J}^{L} \Pi_{L K]} .
$$

The square brackets are alternation in the extreme indices in these brackets. These components satisfy the differential comparisons modulo basis forms $\theta^{I}$ of the space $P_{n}$ :

$$
\Delta K_{J K L}^{I} \cong 0, \quad \Delta K_{I J K}+K_{I J K}^{L} \theta_{L} \cong 0 .
$$

Proposition 3. The centroprojective curvature object $K$ is a tensor containing the subtensor of the affine curvature $K_{J K L}^{I}$. 
We put the affine connection forms $\left(1.4_{1}\right)$ into the structure equations $\left(1.2_{1}\right)$ of the base $P_{n}$

$$
D \theta^{I}=\theta^{J} \wedge \hat{\theta}_{J}^{I}+S_{J K}^{I} \theta^{J} \wedge \theta^{K},
$$

where $S_{J K}^{I}=\Pi_{[J K]}^{I}$ are the components of the affine torsion object of the centroprojective connection. The differential equations $\left(1.5_{1}\right)$ with the symmetry of the forms $\theta_{J K}^{I}(1.3)$ in the lower indices imply the comparisons $\Delta S_{J K}^{I} \cong 0$.

Proposition 4. The bundle $C_{n(n+1)}\left(P_{n}\right)$ of centroprojective frames with given centroprojective connection is the space $C_{n(n+1), n}$ of the centroprojective connection with closed structure equations (1.6), (1.7) which contain the affine torsion tensor $S_{J K}^{I}$ and the centroprojective curvature object $K$. The space $C_{n(n+1), n}$ has the quotient space of an affine connection $L_{n^{2}, n}(1.7),\left(1.6_{1}\right)$ with the torsion $S_{J K}^{I}$ and curvature $K_{J K L}^{I}$ tensors.

According to [5], we can introduce the torsion object (centroprojective torsion object) of the centroprojective connection $S=\left\{S_{J K}^{I}, S_{I J}\right\}$, where $S_{I J}=\Pi_{[I J]}$. The differential equations (1.52) imply

$$
\Delta S_{I J}+S_{I J}^{K} \theta_{K} \cong 0 .
$$

Proposition 5. The centroprojective torsion object $S$ is a tensor containing the affine torsion subtensor $S_{J K}^{I}$.

Definition. The centroprojective connection is an affine symmetric connection (an affine torsion-free connection) if $S_{J K}^{I}=0$. The centroprojective connection is a symmetric connection (a centroprojective torsion-free connection) if $S=0$.

Conclusion 1. The forms $\theta_{J K}^{I}$ (1.3) in the differential equations (1.5 ) for the components of the affine subconnection object $\Pi_{J K}^{I}$ are symmetric forms. Therefore, the affine connection have to be symmetric connection $\left(\Pi_{[J K]}^{I}=0\right)$. There are the symmetric components $\Pi_{J K}^{I}$ in the differential equations $\left(1.5_{2}\right)$ for the components $\Pi_{I J}$ of the object $\Pi$ hence $\Pi_{I J}$ are symmetric components $\left(\Pi_{[I J]}=0\right.$. $)$ Therefore, we can put only symmetric centroprojective connection into the projective group $\mathrm{GP}(n)=C_{n(n+1)}\left(P_{n}\right)$.

\section{FUNDAMENTAL-GROUP CONNECTION ASSOCIATED WITH A SURFACE}

In the projective space $P_{n}$ we shall consider $m$-dimensional surface $S_{m}(1 \leq m<$ $n$ ) as the family of the centered tangent planes $T_{m}$. Let us partition the indices set as:

$$
I=(i, a) ; \quad i, \cdots=\overline{1, m} ; \quad a, \cdots=\overline{m+1, n} .
$$

Let us put the tops $A, A_{i}$ of the moving frame $\left\{A, A_{I}\right\}$ on the tangent plane $T_{m}$ so that the top $A$ coincides with the tangent point. According to (1.1), let us write the 
equations of the surface $S_{m}$ in the form

$$
\begin{gathered}
\theta^{a}=0, \\
\theta_{i}^{a}=\Lambda_{i j}^{a} \theta^{j} .
\end{gathered}
$$

Closing the equations $\left(2.1_{1}\right)$ we obtain $\Lambda_{[i j]}^{a}=0$. Prolonging $\left(2.1_{2}\right)$ we have

$$
\begin{gathered}
\Delta \Lambda_{i j}^{a} \approx 0, \\
\Delta \Lambda_{i j}^{a}=\partial \Lambda_{i j}^{a}+\Lambda_{i j}^{b} \omega_{b}^{a}-\Lambda_{k j}^{a} \omega_{i}^{k}-\Lambda_{i k}^{a} \omega_{j}^{k},
\end{gathered}
$$

where $\partial=\left.d\right|_{S_{m}}, \quad \omega=\left.\theta\right|_{S_{m}}$, the symbol $\approx$ is the comparison modulo basis forms $\theta^{i}$ of the surface $S_{m}$.

Eliminating the principal forms $\theta^{i}, \theta^{a}, \theta_{i}^{a}$ of the equations (2.1) for the surface $S_{m}$ from the structure forms $\theta^{I}, \theta_{J}^{I}, \theta_{I}$ of the projective group $\operatorname{GP}(n)$ we keep the secondary forms. They are called the fibre forms on the surface $S_{m}$. The basis forms $\theta^{i}$ and fiber forms $\omega_{j}^{i}, \omega_{i}, \omega_{b}^{a}, \omega_{a}^{i}, \omega_{a}$ satisfy the structure equations [1,9]

$$
\begin{gathered}
D \theta^{i}=\theta^{j} \wedge \omega_{j}^{i} ; \\
D \omega_{j}^{i}=\omega_{j}^{k} \wedge \omega_{k}^{i}+\theta^{k} \wedge \omega_{j k}^{i}, \\
\omega_{j k}^{i}=\Lambda_{j k}^{a} \omega_{a}^{i}-\delta_{j}^{i} \omega_{k}-\delta_{k}^{i} \omega_{j} ; \\
D \omega_{i}=\omega_{i}^{j} \wedge \omega_{j}+\theta^{i} \wedge \omega_{i j}, \\
\omega_{i j}=\Lambda_{i j}^{a} \omega_{a} ; \\
D \omega_{b}^{a}=\omega_{b}^{c} \wedge \omega_{c}^{a}+\theta^{i} \wedge \omega_{b i}^{a}, \quad \omega_{b i}^{a}=-\Lambda_{i j}^{a} \omega_{b}^{j}-\delta_{b}^{a} \omega_{i} ; \\
D \omega_{a}^{i}=\omega_{a}^{j} \wedge \omega_{j}^{i}+\omega_{a}^{b} \wedge \omega_{b}^{i}+\theta^{j} \wedge \omega_{a j}^{i}, \\
\omega_{a j}^{i}=-\delta_{j}^{i} \omega_{a} ; \\
D \omega_{a}=\omega_{a}^{i} \wedge \omega_{i}+\omega_{a}^{b} \wedge \omega_{b} .
\end{gathered}
$$

We obtain the structure equations (2.3)-(2.8) of the principal bundle $G_{r}\left(S_{m}\right)$ associated with $S_{m}$. The surface $S_{m}$ is a base of the principal bundle $G_{r}\left(S_{m}\right)$. The subgroup of stationarity $G_{r} \subset \mathrm{GP}(n)$ of the centered tangent plane $T_{m}$ is a typical fiber. We have

$$
r=\operatorname{dim} G_{r}=n(n+1)-m(n-m) .
$$

Associated fibering $G_{r}\left(S_{m}\right)$ has 4 simple quotient principal bundles [10] over the base $S_{m}$ with the structure equations:

(1) (2.3), (2.4) - the tangent linear frame fibering $L_{m^{2}}\left(S_{m}\right)$ with the typical fiber $L_{m^{2}}=\mathrm{GL}(m)$, where $\mathrm{GL}(m)$ is linear quotient group acting ineffectively on the bunch of tangent lines passing through the center $A$ (on the quotient space $T_{m-1}=T_{m} / A$ ); 
(2) (2.3)-(2.5) - the tangent centroprojective frame fibering $C_{m(m+1)}\left(S_{m}\right)$ with the typical fiber $C_{m(m+1)}=G A^{*}(m)$, where $G A^{*}(m)$ is centroprojective (coaffine) quotient group acting effectively on the centered tangent plane $T_{m}$;

(3) (2.3), (2.6) - the normal linear frame fibering $L_{(n-m)^{2}}\left(S_{m}\right)$ with the typical fiber $L_{(n-m)^{2}}=\mathrm{GL}(n-m)$, where $\mathrm{GL}(n-m)$ is linear quotient group acting ineffectively in the $(n-m-1)$-dimensional projective space $P_{n-m-1}=$ $P_{n} / T_{m}$ (see, e.g., [1]);

(4) (2.3), (2.4), (2.6), (2.7) - the fibering $H_{m^{2}-m n+n^{2}}\left(S_{m}\right)$ with the typical fiber $H_{m^{2}-m n+n^{2}}$. This fiber is:

(a) stationarity subgroup for the centered tangent plane to $m$-dimensional surface of $n$-dimensional affine space $A_{n}$,

(b) stationarity subgroup for the tangent straight lines subbunch on the straight line bunch of the space $P_{n}$ with the center $A$,

(c) stationarity subgroup for the quotient plane $T_{m-1}=T_{m} / A$ in $P_{n} / A$,

(d) an affine quotient group [11] of the projective subgroup $G_{r}$.

According to the Laptev-Lumiste method, a connection in the principal bundle $G_{r}\left(S_{m}\right)$ is defined by the forms

$$
\begin{gathered}
\tilde{\omega}_{j}^{i}=\omega_{j}^{i}-\Gamma_{j k}^{i} \theta^{k}, \quad \tilde{\omega}_{i}=\omega_{i}-\Gamma_{i j} \theta^{j}, \\
\tilde{\omega}_{b}^{a}=\omega_{b}^{a}-\Gamma_{b i}^{a} \theta^{i}, \quad \tilde{\omega}_{a}^{i}=\omega_{a}^{i}-\Gamma_{a j}^{i} \theta^{j}, \quad \tilde{\omega}_{a}=\omega_{a}-\Gamma_{a i} \theta^{i},
\end{gathered}
$$

and the components of the fundamental-group connection object

$$
\Gamma=\left\{\Gamma_{j k}^{i}, \Gamma_{i j}, \Gamma_{b i}^{a}, \Gamma_{a j}^{i}, \Gamma_{a i}\right\}
$$

satisfy the differential equations [9]

$$
\begin{aligned}
\Delta \Gamma_{j k}^{i}+\omega_{j k}^{i} & =\Gamma_{j k l}^{i} \theta^{l}, \\
\Delta \Gamma_{i j}+\Gamma_{i j}^{k} \omega_{k}+\omega_{i j} & =\Gamma_{i j k} \theta^{k}, \\
\Delta \Gamma_{b i}^{a}+\omega_{b i}^{a} & =\Gamma_{b i j}^{a} \theta^{j}, \\
\Delta \Gamma_{a j}^{i}+\Gamma_{a j}^{b} \omega_{b}^{i}-\Gamma_{k j}^{i} \omega_{a}^{k}+\omega_{a j}^{i} & =\Gamma_{a j k}^{i} \theta^{k}, \\
\Delta \Gamma_{a i}+\Gamma_{a i}^{j} \omega_{j}+\Gamma_{a i}^{b} \omega_{b}-\Gamma_{j i} \omega_{a}^{j} & =\Gamma_{a i j} \theta^{j} .
\end{aligned}
$$

The connection object $\Gamma$ has 4 simple subobjects [10]: $\Gamma_{j k}^{i}$ - the tangent affine connection object, $\left\{\Gamma_{j k}^{i}, \Gamma_{i j}\right\}$ - the tangent centroprojective connection object, $\Gamma_{b i}^{a}$ - the normal linear connection object, $\left\{\Gamma_{j k}^{i}, \Gamma_{b i}^{a}, \Gamma_{a j}^{i}\right\}$ - affine-group connection object. These subobjects give the fundamental-group connections in the corresponding quotient fiberings of the associated fibering $G_{r}\left(S_{m}\right)$.

Conclusion 2. The forms $\omega_{j k}^{i}\left(2.4_{2}\right), \omega_{i j}\left(2.5_{2}\right)$ from the differential equations $\left(2.10_{1}\right)$ and $\left(2.10_{2}\right)$ for the components of the centroprojective connection object 
$\left\{\Gamma_{j k}^{i}, \Gamma_{i j}\right\}$ are symmetric forms. Therefore, on the surface $S_{m}$ we can consider only connection centroprojective torsion-free: $\Gamma_{[j k]}^{i}=0, \Gamma_{[i j]}=0$.

The fundamental-group connection forms (2.9) satisfy the structure equations

$$
\begin{gathered}
D \tilde{\omega}_{j}^{i}=\tilde{\omega}_{j}^{k} \wedge \tilde{\omega}_{k}^{i}+R_{j k l}^{i} \theta^{k} \wedge \theta^{l}, \quad D \tilde{\omega}_{i}=\tilde{\omega}_{i}^{j} \wedge \tilde{\omega}_{j}+R_{i j k} \theta^{j} \wedge \theta^{k}, \\
D \tilde{\omega}_{b}^{a}=\tilde{\omega}_{b}^{c} \wedge \tilde{\omega}_{c}^{a}+R_{b i j}^{a} \theta^{i} \wedge \theta^{j}, \quad D \tilde{\omega}_{a}^{i}=\tilde{\omega}_{a}^{j} \wedge \tilde{\omega}_{j}^{i}+\tilde{\omega}_{a}^{b} \wedge \tilde{\omega}_{b}^{i}+R_{a j k}^{i} \theta^{j} \wedge \theta^{k}, \\
D \tilde{\omega}_{a}=\tilde{\omega}_{a}^{i} \wedge \tilde{\omega}_{i}+\tilde{\omega}_{a}^{b} \wedge \tilde{\omega}_{b}+R_{a i j} \theta^{i} \wedge \theta^{j},
\end{gathered}
$$

where the components of curvature object

$$
R=\left\{R_{j k l}^{i}, R_{i j k}, R_{b i j}^{a}, R_{a j k}^{i}, R_{a i j}\right\}
$$

of the fundamental-group connections $\Gamma$ are expressed by the formulas

$$
\begin{gathered}
R_{j k l}^{i}=\Gamma_{j[k l]}^{i}-\Gamma_{j[k}^{m} \Gamma_{m l]}^{i}, \quad R_{i j k}=\Gamma_{i[j k]}-\Gamma_{i[j}^{l} \Gamma_{l k]}, \quad R_{b i j}^{a}=\Gamma_{b[i j]}^{a}-\Gamma_{b[i}^{c} \Gamma_{c j]}^{a}, \\
R_{a j k}^{i}=\Gamma_{a[j k]}^{i}-\Gamma_{a[j}^{l} \Gamma_{l k]}^{i}-\Gamma_{a[j}^{b} \Gamma_{b k]}^{i}, \quad R_{a i j}=\Gamma_{a[i j]}-\Gamma_{a[i}^{k} \Gamma_{k j]}-\Gamma_{a[i}^{b} \Gamma_{b j]} .
\end{gathered}
$$

These components satisfy the differential comparisons [10]

$$
\begin{gathered}
\Delta R_{j k l}^{i} \approx 0, \quad \Delta R_{i j k}+R_{i j k}^{l} \omega_{l} \approx 0, \quad \Delta R_{b i j}^{a} \approx 0, \\
\Delta R_{a j k}^{i}-R_{l j k}^{i} \omega_{a}^{l}+R_{a j k}^{b} \omega_{b}^{i} \approx 0, \quad \Delta R_{a i j}+R_{a i j}^{k} \omega_{k}+R_{a i j}^{b} \omega_{b}-R_{k i j} \omega_{a}^{k} \approx 0 .
\end{gathered}
$$

Theorem 1. The curvature object $R$ of the fundamental-group connection $\Gamma$ is a tensor containing:

(1) the curvature tensor $R_{j k l}^{i}$ of the tangent affine connection $\Gamma_{j k}^{i}$,

(2) the curvature tensor $\left\{R_{j k l}^{i}, R_{i j k}\right\}$ of the tangent centroprojective connection $\left\{\Gamma_{j k}^{i}, \Gamma_{i j}\right\}$,

(3) the curvature tensor $R_{b i j}^{a}$ of the normal linear connection $\Gamma_{b i}^{a}$,

(4) the curvature tensor $\left\{R_{j k l}^{i}, R_{b i j}^{a}, R_{a j k}^{i}\right\}$ of the affine-group connection $\left\{\Gamma_{j k}^{i}\right.$, $\left.\Gamma_{b i}^{a}, \Gamma_{a j}^{i}\right\}$.

\section{REDUCTION OF THE CENTROPROJECTIVE CONNECTION}

We define a symmetric centroprojective connection object $\Pi=\left\{\Pi_{J K}^{I}, \Pi_{I J}\right\}$. By the partition of each index into two indices the object $\Pi$ will consist from the following essential components

$$
\Pi=\left\{\Pi_{j k}^{i}, \Pi_{i j}^{a}, \Pi_{a j}^{i}, \Pi_{b i}^{a}, \Pi_{a b}^{i}, \Pi_{b c}^{a}, \Pi_{i j}, \Pi_{a i}, \Pi_{a b}\right\} .
$$

In the differential equations (1.5) for the components of the object $\Pi$ we expand the action of the operator $\Delta$ and partition the indices. We take the equations (2.1) of 
the surface $S_{m}$, apply the operator $\Delta$ to the subobjects and write result in the comparisons form for the components analogical to the components of the fundamental object $\Lambda_{i j}^{a}$ and connection object $\Gamma$ :

$$
\begin{aligned}
\Delta \bar{\Pi}_{i j}^{a} & \approx 0, \\
\Delta \bar{\Pi}_{j k}^{i}+\bar{\Pi}_{j k}^{a} \omega_{a}^{i}-\delta_{j}^{i} \omega_{k}-\delta_{k}^{i} \omega_{j} & \approx 0, \\
\Delta \bar{\Pi}_{b i}^{a}-\bar{\Pi}_{j i}^{a} \omega_{b}^{j}-\delta_{b}^{a} \omega_{i} & \approx 0, \\
\Delta \bar{\Pi}_{a j}^{i}+\bar{\Pi}_{a j}^{b} \omega_{b}^{i}-\bar{\Pi}_{k j}^{i} \omega_{a}^{k}-\delta_{j}^{i} \omega_{a} & \approx 0, \\
\Delta \bar{\Pi}_{i j}+\bar{\Pi}_{i j}^{k} \omega_{k}+\bar{\Pi}_{i j}^{a} \omega_{a} & \approx 0, \\
\Delta \bar{\Pi}_{a i}-\bar{\Pi}_{j i} \omega_{a}^{j}+\bar{\Pi}_{a i}^{j} \omega_{j}+\bar{\Pi}_{a i}^{b} \omega_{b} & \approx 0 .
\end{aligned}
$$

In (3.1) $\bar{\Pi}=\left.\Pi\right|_{S_{m}}$. From the coincidence of the differential comparisons (2.21) and $\left(3.1_{1}\right)$ we have

Lemma 1. The subobject $\Pi_{i j}^{a}$ (of the centroprojective connection object $\Pi$ without the torsion) restricted to the surface $S_{m}$ is identified with the fundamental object $\Lambda_{i j}^{a}$ :

$$
\bar{\Pi}_{i j}^{a}=\Lambda_{i j}^{a} \text {. }
$$

Comparing the differential equations (2.10) with the forms $\left(2.4_{2}\right)-\left(2.7_{2}\right)$ and the comparisons $\left(3.1_{2}\right)-\left(3.1_{6}\right)$ by means of Lemma 1 we obtain the equalities

$$
\Gamma_{j k}^{i}=\bar{\Pi}_{j k}^{i}, \quad \Gamma_{i j}=\bar{\Pi}_{i j}, \quad \Gamma_{b i}^{a}=\bar{\Pi}_{b i}^{a}, \quad \Gamma_{a j}^{i}=\bar{\Pi}_{a j}^{i}, \quad \Gamma_{a i}=\bar{\Pi}_{a i} .
$$

Theorem 2. The symmetric centroprojective connection on the projective group $\mathrm{GP}(n)$ effectively acting in projective space $P_{n}$ is reduced to the fundamental-group connection on the surface $S_{m}$ of the space $P_{n}$.

Remark 1. The components $\Pi_{a b}^{i}, \Pi_{b c}^{a}, \Pi_{a b}$ of the connection object $\Pi$ are not used (compare [7,12]) for the reduction of the object $\Pi$ to the object $\Gamma$.

\section{REFERENCES}

[1] M. A. Akivis and V. V. Goldberg, Projective differential geometry of submanifolds. Elsevier, 1993.

[2] A. V. Chakmazjan, Normal connection in geometry of submanifolds (in Russian). Erevan, 1990.

[3] S. Kobayashi, Transformation groups in differential geometry. Springer Verlag, 1972.

[4] G. F. Laptev, "Manifolds immersioned into generalized spaces (in Russian)," in Proceedings of 4-th All-Union math. congress, vol. 2. Izdat. Nauka, Leningrad, 1964, pp. 226-233.

[5] V. G. Lemlein, "Local centroprojective spaces and connections in a differentiable manifold (in Russian)," Litovsk. Math. Sb., vol. 4, no. 1, pp. 41-132, 1964.

[6] Y. G. Lumiste, "Connections on homogeneous fiberings (in Russian)," Math. Sb., vol. 69, no. 3, pp. 434-469, 1966.

[7] O. M. Omelyan and Y. I. Shevchenko, "Reductions of the object and the affine torsion tensor of a centroprojective connection on a distribution of planes," Math. Notes, vol. 84, no. 1-2, pp. 100-107, 2008. 
[8] Y. I. Shevchenko, "Laptev's and Lumiste's ways of the giving a connection in the principal fiber bundle," Proceedings of the International Geometry Center, vol. 3, no. 1, pp. 46-53, 2010.

[9] Y. I. Shevchenko, "About the clothings of the multidimensional surface of the projective space (in Russian)," Differential geometry of manifolds of figures, vol. 8, pp. 135-150, 1977.

[10] Y. I. Shevchenko, Framings of centroprojective manifolds (in Russian). Kaliningrad, 2000.

[11] Y. I. Shevchenko, "Affine, coaffine and linear quotient groups in subgroups of the projective group (in Russian)," in Problems of mathematical and physical sciences, Kaliningrad, 2002, pp. 38-39.

[12] Y. I. Shevchenko, Connections associated with a distribution of planes in the projective space (in Russian). Kaliningrad, 2009.

Authors' addresses

\section{Jury Shevchenko}

Immanuel Kant Baltic Federal University, Department of Fundamental Mathematics, 14 A. Nevsky St., 236041 Kaliningrad, Russia

Elena Skrydlova

Immanuel Kant Baltic Federal University, Department of Computer Safety, 14 A. Nevsky St., 236041 Kaliningrad, Russia

E-mail address: ESkrydlova@kantiana.ru 